\title{
Irradiation models for ULXs and fits to HST observations of NGC 4559 X-7
}

\author{
Chris Copperwheat ${ }^{1}$, Mark Cropper ${ }^{1}$, \\ Roberto Soria ${ }^{1,2}$ and Kinwah $\mathbf{W u}^{1}$ \\ ${ }^{1}$ Mullard Space Science Laboratory, University College London, Holmbury St. Mary, Dorking, \\ Surrey, RH5 6NT, UK \\ ${ }^{2}$ Harvard-Smithsonian Center for Astrophysics, 60 Garden Street, Cambridge, MA 02138, USA \\ email: cmc@mssl.ucl.ac.uk
}

\begin{abstract}
We detail a model we have created to describe the optical emission from a ULX in terms of an irradiated companion star and disk. We apply this model to optical observations of ULX X-7 in NGC 4559. We revise the parameters of the companion star in this system to be older, less massive and of a later spectral type than previously reported. We find the black hole to be of a few hundred solar masses at most.
\end{abstract}

Keywords. accretion, accretion disks, black hole physics, X-rays: galaxies, X-rays: stars.

\section{Introduction}

Ultra-luminous X-ray sources (ULXs) are point-like, non-nuclear sources with apparent isotropic luminosities greater than $10^{39} \mathrm{ergs} \mathrm{s}^{-1}$. The brightest sources have X-ray luminosities in excess of $10^{40} \mathrm{ergs} \mathrm{s}^{-1}$, implying bolometric luminosities of order $10^{41} \mathrm{ergs} \mathrm{s}^{-1}$. This is significantly larger than the Eddington luminosity limit for an accreting stellarmass black hole (BH). The nature of these objects is still unclear. The high luminosities may be a result of beaming towards the observer. Alternatively, the accreting object may be an intermediate mass black hole $(\mathrm{IMBH})$ with mass $50-10000 M_{\odot}$. We have created a model describing the optical emission from ULXs. Our model is useful to identify optical counterparts. Our study has shown that optical observations are also powerful tools, as X-ray observations, to determine the properties and hence the nature of ULX.

\section{Model}

We consider a binary model, with the compact object accreting material from a companion star. We assume the X-ray emission is isotropic, and hence take the IMBH interpretation as a starting point. The brightest ULXs require an accretion rate greater than that which could be supplied by a stellar wind, so we assume the matter is transferred onto the compact object through Roche lobe overflow. We constrain the geometry of the system so that the companion star is filling its Roche lobe. This constraint necessitates a small binary separation and a large companion star. A large amount of X-ray flux will be incident on the surface of this star, and the optical/IR characteristics of this star will be modified. The irradiation will induce intensity and colour shifts compared to normal stars, which we use as a diagnostic. We assume the system is in a quasi-steady state, and the irradiated surfaces are in thermal, radiative and hydrostatic equilibrium. We consider the effects of radiative transport and radiative equilibrium in the irradiated surface of the star and an irradiated accretion disk. We consider a plane-parallel model and adopt the radiative transport formulation of Milne (1926) and Wu et al. (2001) to describe the 

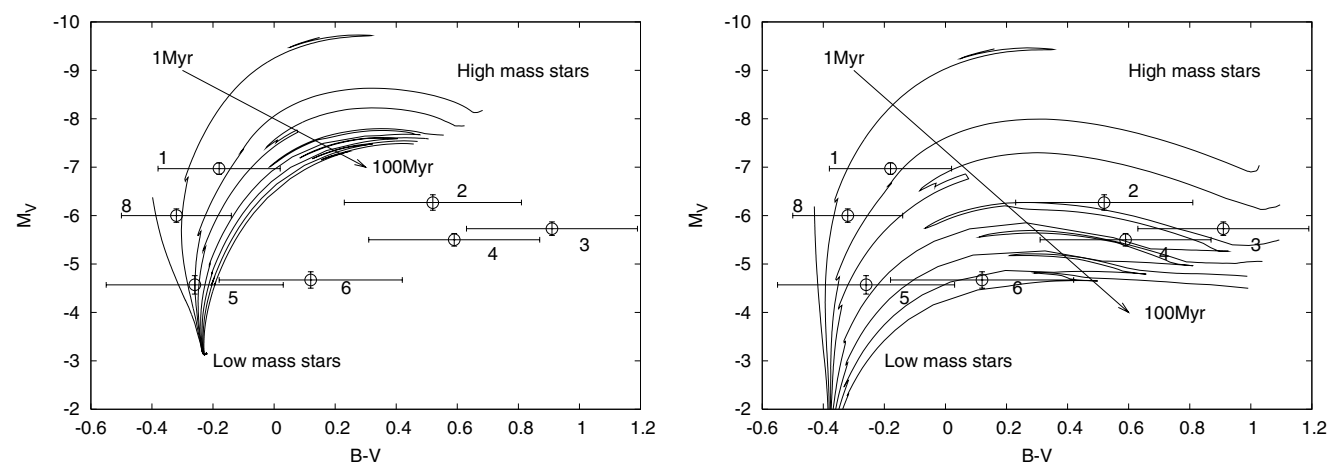

Figure 1. Colour - magnitude diagrams showing the Geneva isochrones modified to include our irradiation model. In the left panel we use an inclination of $\cos i=0.5$ and a $\mathrm{BH}$ mass of $100 M_{\odot}$. In the right panel we use $\cos i=0.0$ and a $\mathrm{BH}$ mass of $1000 M_{\odot}$. If we require candidates 2,3 and 4 to fit with our model, then this combination of a low inclination and a high BH mass is necessary.

heated surface. We determine the total emergent radiation from a distorted, Roche lobe filling star numerically. We do the same for the disk, using a thin disk geometry.

The model is described more completely in Copperwheat et al. (2005).

\section{Application to ULX X-7 in NGC 4559}

A ULX with $L_{x} \simeq 10^{40} \mathrm{ergs} \mathrm{s}^{-1}$ was found in the late type spiral galaxy NGC 4559 $(\mathrm{d}=10 \mathrm{Mpc})$. HST observations revealed eight candidates for the optical counterpart (Soria et al. 2005) We apply our irradiation model to stars evolved according to the Geneva isochrones of Lejeune \& Schaerer (2001) and then compare these modified isochrones to the observations. We do this for a range of orientations, inclinations and BH masses (Figure 1). We find that some candidates are consistent with our model irradiated star and disk only when we use a very low inclination and a very high BH mass. If the inclination is such that the optical emission contains an appreciable disk component, we find that these candidates $(2,3$ and 4$)$ do not fit with any $\mathrm{BH}$ and star combination.

Soria et al. (2005) concluded that candidate 1 was the most likely counterpart to the ULX. The other candidates were found to be consistent with the unperturbed isochrones for stars with masses $10-15 M_{\odot}$ and ages of approximately $20 \mathrm{Myr}$. If we assume the companion star is also this age and that candidate 1 is indeed the counterpart, then by comparing the $20 \mathrm{Myr}$ isochrone with the observed magnitudes we find the mass of the companion star to be $11-12 M_{\odot}$ and the radius to be $40-50 R_{\odot}$. We find also that these assumptions imply an upper bound on the $\mathrm{BH}$ mass range of a few hundred solar masses.

\section{References}

Copperwheat, C. M., Cropper, M., Soria, R. \& Wu, K. 2005, MNRAS, 362, 79

Lejeune, T. \& Schaerer, D., 2001, A\&A, 366, 538

Milne, E. A. 1926, MNRAS, 87, 43

Soria, R., Cropper, M., Pakull, M., Mushotzky, R. \& Wu, K., 2005, MNRAS, 356, 12

Wu, K., Soria, R., Hunstead, R. W. \& Johnston, H.M. 2001, MNRAS, 320, 177 